\title{
Perfil do risco de óbito de crianças menores de um ano residentes em localidade do Estado de São Paulo, Brasil, 1987*
}

\author{
Death risk profile of children under one year of age in an interior town of \\ S. Paulo State, Brazil, in 1987
}

Carlos Alberto Macharelli**, Luiz Roberto de Oliveira**

\begin{abstract}
MACHARELLI, C. A. \& OLIVEIRA, L. R. Perfil do risco de óbito de crianças menores de um ano residentes em localidade do Estado de São Paulo, Brasil, 1987. Rev. Saúde públ., S. Paulo, 25: 1218, 1991. Estudou-se a mortalidade de crianças menores de um ano de idade residentes no Município de Botucatu, SP (Brasil), em 1987. Tentando estabelecer o perfil de risco desses óbitos, tendo como seu principal responsável a desigualdade social, calculou-se o risco adicional (RA) em função de algumas variáveis usando a metodologia de estudos de caso-controle. O resultado obtido foi um RA de óbito de 15,58 para gestação pré-termo, 11,63 para o baixo peso ao nascer, 8,50 para inexistência de água intradomiciliar e 4,04 para escolaridade materna insuficiente. Verificou-se existir importante desigualdade entre as famílias das crianças que morreram e as das que sobreviveram, sugerindo que a melhor estratégia para enfrentar o excesso de mortalidade infantil residiria na melhoria sócio-econômica, isto é, todas as famnias deveriam ter a mesma capacidade de consumir os bens e serviços em igual quantidade e qualidade.
\end{abstract}

Descritores: Mortalidade infantil. Risco. Fatores sócio-econômicos.

\section{Introdução}

O estudo das caractcrísticas da mortalidade na infância, levado a efcito nas Américas cm $1972^{22}$, mostrou que os principais problemas relacionados aos óbitos até os 5 anos de vida eram, além das doenças infecciosas e da deficiência nutricional, alguns fatores relacionados com as condiçōes maternas, tais como: complicações da gravidez, condições do parto, desmame precoce, condições de moradia, grau de instrução materna, além de outros.

Posteriormente ao estudo acima citado, vários autores brasileiros aprofundaram cstudos sobre a mortalidade infantil, tanto em nível do Brasil $^{26}$, como de cada Estado ${ }^{18}$. No presente trabalho scrão ressaltados apenas alguns desses estudos: Leser ${ }^{11}$, que estudou o crescimento populacional na Grande São Paulo entre 1950 e 1970 e scus reflexos nas condições de saúde pública; Montciro ${ }^{17}$, que fez importantes observações $\mathrm{em}$ rclação à distribuição diferencial da mortalidade infantil entre determinadas áreas do Município de São Paulo e ofera de

* Publicaçãofinanciada pcla FAPESP.

* Departamento de Medicina Legal e Medicina em Saúde Pública da Faculdade de Medicina de Botucatu da Universidade Estadual Paulista. Campus de Botucalu, SP Brasil.

Separata/Reprints: C. A. Macharclli - Rubião Júnior 18610 - Botucatu, SP - Brasil. serviços públicos de saúde. Posteriormente, Montciro e col. ${ }^{19}$ rclacionaram em seus estudos a mortalidade infantil com a renda familiar e o abastecimento de água. Um estudo mais recente, realizado por Amigo e col. ${ }^{1}$ na cidade de Recife, sobre mortalidade em menores de 5 anos, chega às seguintes conclusões: "as associações estudadas refletem a importância das variáveis sócio-econômicas, especialmente habitação e instrução materna. Já as análises sócio-demográficas não permitem, neste tipo de estudo, opinião definitiva sobre sua influência c associações".

O que se obscrva em muitos trabalhos publicados é a crescente preocupacão dos autores em tenLar relacionar a mortalidade infantil com "fatores sócio-econômicos" e a implicaçāo deles sobre ela. Olivcira e col. ${ }^{21}$ destacam "a parcialidade de análise destes estudós que, embora lidando com variávcis de naturcza sócio-cconômica, não as referenciam com a estrutura social"; isto implica, segundo os cilados autores, conclusões e recomendações que levadas à prálica pelos "programas oficials de saúde, têm-se mostrado incapazes de dar conta da efcliva melhoria das condições de saúde das populações latino-americanas, após uma década de tentativas". No entanto, já existem hoje, especialmente na literatura latino-amcricana, publicações que procuram dar mais conseqüência ao cstudo das relações entre economia, saúde e política. Nesses estudos os autores constroem um 
referencial de análise, capaz de estabclecer a conexão entre as manifestações individuais e particulares do processo saúde-doença com as condições de existência das coletividades, estas vistas como organizaçōes sociais heterogêneas produzidas pclo modo de produção vigente $e^{3,4,5}$. Nesta linha de preocupação, Oliveira ${ }^{21}$ procurou construir o quadro explicativo da desnutrição protéico-energética e discute vários "fatores sócio-econômicos" e os organiza na ordem de precedência, tendo num extremo a condição individual "desnutrição e óbilo" e, no outro, o modo de produção. O processo saúdedoença individual e social é visto como consequiência da capacidade de consumo individual $\mathrm{e}$ social de bens e serviços que, por sua vez, decorre da posicão ocupada pelos indivíduos e seus pares na estrutura produtiva. Neste sentido, os rendimentos ganham precedência sobre outras características mensuráveis em inquéritos como o realizado pelo autor, pois em conjunturas urbanas da sociedade capitalista é com dinheiro que se $\mathrm{tcm}$ acesso à moradia, às vestes, aos alimentos, à cscola e à assistência à saúdc. O autor destaca ainda o papel do nível de instrução, nessas mesmas conjunturas urbanas, como indicador da condição social, pois é com ele que se tcm acesso ao emprego e a melhores posiçōes na estrutura produliva. Com essas abordagens ${ }^{3,4,5,20}$ os cstudos apontam para transformações mais profundas na realidade social, procurando acabar com as desigualdades.

O trabalho de Fischmann e Guimarãcs? alćm de reforçar as conclusões de Oliveira ${ }^{20,21}$, chega às conclusões seguintes: "Algumas das cifras são alarmantes e localizam o problema em termos de prioridades. A par do problema social que acompanha as piores tuxas, chama a atenção a necessidade de se verificar qual a acessibilidade e a qualidade de assistência à saúde posta à disposição da população favelada, pois muitas dessas causas podem ser reduzidas com cuidados médicos adcquados". Os citados autores utilizam nesse trabalho o enfoque de risco. Esse enfoque vem sendo usado em vários países e regiõcs, e inúmeros são os trabalhos publicados sobre o assunto $\mathrm{em}$ nível mundial. No entanto, muito pouco $\mathrm{tcm}$ sido publicado no Brasil.

O presente trabalho é uma tentativa de estabclecimento do perfil de risco de óbito para crianças menores que um ano de idade, levando-se cm conta a desigualdade social como fontc geradora de condições desiguais de sobrevivência dessas crianças. A desigualdade social é um componente essencial da estrutura econômico-social c sc cvidencia por graus diferentes de consumo de bens e serviços ${ }^{14}$. Partindo do pressuposto que os scrviços de saúde exercem papel imporlante $\mathrm{cm}$ relação à morbi-mortalidade da população, cspe- cialmente em centros de estágio de desenvolvimento cconômico-social mais avançado, como é o caso dos municípios de São Paulo ${ }^{18}$, Pelotas ${ }^{25}$, Porto Alegre? ${ }^{7}$ com os quais Botucatu - SP guarda uma ccrta scmelhança, cabcria produzir subsídios para que serviços de saúde possam ser organizados a partir do enfoque de risco.

O objetivo do presente estudo é, portanto, estabelccer o perfil de risco de óbito em crianças com menos de um ano de idade, determinado pelas desigualdades observadas perante 0 processo de reprodução biológica e social das famílias.

\section{Metodologia}

O cstudo foi realizado em 1987 no Município de Botucatu, Estado de São Paulo, que contava com uma população estimada de 73.951 habitantes*, com $95 \%$ de urbanização. Bolucatu é um município antigo do interior paulista, que conta com inlra-estrutura urbana razoavelmente bem desenvolvida, o que o faz centro regional de comércio e scrviços. A industrialização é incipiente e vem se fazendo há pouco mais de uma década.

O levantamento dos óbitos de crianças menores que cinco anos $c$ dos nascimentos de crianças residentes no Município de Botucalu foi feito para os anos de 1983, 1984 c 1985. Foram obtidos os seguintes valores médios anuais: 63 óbitos/ano e 1.684 nascidos vivos/ano; isto equivale aos valores médios mensais de 5 óbitos e 140 nascidos vivos*.

A colcta dos dados foi feita dos registros vitais, isto $\mathfrak{e}$, dos alestados de óbilos e registros de nascimentos, nos cartórios existentes no Município de Botucatu. Foram colhidos dados somente de crianças menores de um ano, nascidas e residentes cm Bolucatu e falecidas no ano de 1987.

Para cumprir os objetivos propostos, foi realizado um estudo do tipo caso-controle ${ }^{12,15}$. Oplou-se por esse lipo de cstudo por apresentar, alćm das vantagens já conhecidas, como a de ser menos oneroso c propiciar resultados em espaço de $\mathrm{tcm}$ po menor, c de poder ser realizado com grupos numericamente pequenos e permitir estimar o risco relativo.

Foram ulilizados os conccitos de risco, fator de risco e enfoque de risco ${ }^{23}$; risco é a medida de probabilidade de ocorrência; e fator de risco é a caractcrística, ou circunstância de indivíduos ou grupos, que se associa ao aumento da probabilidade de

\footnotetext{
* Ionte: Seção de Epidemologia e Estatística do Centro de Saúde Escola de Bolucatu: arquivos de nascimentos e óbitos.
} 
ocorrência de um evento não desejado. Considerou-se: Caso, todo óbito de criança menor que um ano, residente no Município de Bolucalu-SP, ocorrido a partir de janeiro a dczembro de 1987; e Controle, toda criança nascida viva, residente no Município de Botucatu-SP, do mesmo sexo e idade do caso. Dentro dessas características foi tomada a criança que nasceu no mesmo dia do caso. A decisão entre vários possíveis controles fcz-se pelo horário de nascimento. Não havendo, tomaram-se inicialmente os nascidos no dia imediatamente posterior e, na ausência desses, os nascidos no dia imediatamente anterior, obedecendo-se o critério de horário de nascimento.

Utilizou-se como instrumento de coleta de dados um questionário aplicado às mães dos casos e dos controles em seus domicílios. Elaborou-se manual no qual se definiu cada categoria utilizada para garantir a padronização do preenchimento dos questionários pelos entrevistadores.

O questionário foi subdividido $\mathrm{cm} 5$ partes: identificação pessoal; condiçōes de nascimento; composição familiar; condições de moradia; assistência médica/morbidade.

Os resultados foram analisados de acordo com as seguintes caractcrísticas:

Classe Social, segundo Barros': subproletariado; proletariado propriamente dito; pequena burgucsia; burguesia gerencial; burguesia empresarial.

Renda "per capila": foram usados os valores do salário mínimo e da renda familiar vigentes no dia da entrevista; com eles foram calculadas as rendas "per capita" familiares, em salários mínimos: $<0,5$ salário mínimo $\geq 0,5$ salário mínimo.

Condições de moradia: foram considerados o número de janelas das habilações como indicador da área residencial e o sancamento básico, ambos como estimativas da capacidade de consumo de bens básicos da familia.

- $n^{2}$ de janelas: 1- 4; e 5-10.

- saneamento básico, segundo, Vculer e Simōes ${ }^{24}$ : abastecimento de água e rede de esgotos: adequado e inadequado.

Escolaridade materna : $<4^{\text {a }}$ sćric (inclui nenhuma), $\geq 4^{a}$ séric (inclui nível superior)

Condições de nascimento como expressão biológica do processo de reprodução:

- Duração da gestação: pré-tcrmo

- Peso ao nascer: $<2.500 \mathrm{~g}$

$$
\text { a termo }
$$

$$
\geq 2.500 \mathrm{~g}
$$

Construídas as tabclas $2 \times 2$, calculou-se o QuiQuadrado $\left(X^{2}\right)$ para cada uma delas. O Risco Adicional (RA) e seu Intervalo de Confiança (IC) foram calculados, segundo Micttinen ${ }^{15}$, somente para as associações nas quais o $X^{2}$ resultou significativo para $\alpha=0,05$.

\section{Descrição dos óbitos estudados}

Durante o ano de 1987 ocorreram em Botucatu - SP, 47 óbitos de crianças menores que um ano, dos quais foram csludados 36 , havendo pois uma perda de $23,4 \%$. Tal perda ocorreu devido a não localização das famílias nos endereçoes que constavam das declarações de óbitos. Foram verificadas perdas também entre os controles; no entanto, efcluou-se reposição segundo o critério já descrito de escolha de controles, o que cvitou prejuízos maiores ao grupo estudado. O confronto do grupo de casos perdidos (11 crianças) com o grupo estudado (34 crianças) resultou $\mathrm{cm}$ igualdade estatística quanto às distribuições por scxo, idade, local de residência e causas de óbito. Neste estudo das pcrdas foi usado o Teste de Goodman ${ }^{8}$ para comparações entre proporçõcs multinomiais e um $\alpha=0,05$. Para cada caso foi estudado um controle, configurando-se um conjunto cuja distribuição por sexo e idade encontra-se na Tabcla 1.

Tabela 1. Distribuição dos óbitos (casos) ocorridos abaixo de um ano, segundo sexo e idade em

\begin{tabular}{|c|c|c|c|}
\hline \multirow{2}{*}{ ldade } & \multicolumn{2}{|c|}{ Sexo } & \multirow{2}{*}{ Total $(\%)$} \\
\hline & Masculino & Feminino & \\
\hline$<28$ dias & 15 & 8 & $23(63,9)$ \\
\hline $28 \mathrm{~d}-1$ ano & 9 & 4 & $13(36,1)$ \\
\hline \multirow[t]{2}{*}{ Total } & 24 & 12 & 36 \\
\hline & $(66,7)$ & $(33,3)$ & $(100)$ \\
\hline
\end{tabular}
Botucatu-SP, 1987.

Na distribuição dos óbitos observa-se predomínio do sexo masculino sobre of feminino (quase o dobro). Isso é cxplicado pclo fáto da "razão da masculinidade ao nascer" de Botucalu, cm 1987, ter sido de 1.125. Esse lato, por si só, já scria capaz de assegurar o predomínio do sexo masculino entre os óbilos. No entanto, além dele, observou-se neste mesmo ano um "coeficiente de mortalidade infantil" diferenciado para os dois scxos: 31,78 para o masculino e 20,26 para o femino. 
Tabela 2. Distribuição dos óbitos ocorridos abaixo de um ano, segundo a causa básica*, Botucatu-SP, 1987.

\begin{tabular}{|c|c|c|}
\hline Causa Básica & $\mathrm{N}^{2}$ & $(\%)$ \\
\hline $\begin{array}{l}\text { I. Afecçōes do periodo perinatal } \\
\text { II. Moléstias Infecciosas } \\
\text { III. Anomalias Congênitas } \\
\text { IV. Causa Indeterminada } \\
\text { V. Causas Externas }\end{array}$ & $\begin{array}{r}18 \\
10 \\
5 \\
2 \\
1\end{array}$ & $\begin{array}{r}(50,0) \\
(27,8) \\
(13,9) \\
(5,5) \\
(2,8)\end{array}$ \\
\hline Total & 36 & $(100,0)$ \\
\hline
\end{tabular}

$\mathrm{Na}$ distribuição dos óbitos por faixa etária observa-se que a participação proporcional dos óbitos neonatais ( $<28$ dias) e tardios ( $28 \mathrm{~d}-1$ ano) é, respectivamente, de $60,5 \%$ e $39,5 \%$, pondo em evidência os períodos pré e perinalais como principais geradores de riscos de óbilos infantis. A participação do período infantil tardio, cujos riscos são mais ligados às condições de vida, é menor. Essa situação de risco fica melhor conhecida mediante a consideração do perfil de causas de óbito que se vê na Tabcla 2.

\section{Resultados e discussão}

As distribuições de casos e controles segundo classe social, renda "per capila", número de janelas da habitação e destino dado aos dejetos (esgotos) não revelaram associações estatisticamentc significativas. Cabe enfatizar, no cntanto, que a distribuição por classe social apontou uma tendência à associação entre "subproletariado" e óbito, com um $\mathrm{X}^{2}$ próximo da significação cstalística.

\section{Peso ao Nascer}

Sabe-se que crianças nascidas de gestação a termo com peso abaixo de $2.500 \mathrm{~g}$ são consideradas de "baixo peso ao nascer", ou desnutridas intraútero. Muitos trabalhos sobre mortalidade inlantil têm mostrado considerável associação entre óbito e baixo peso ao nascer, principalmente no período neonatal $1^{9,16}$.

O que verificamos no presente trabalho não é diferente disto. Em Botucatu, no ano de 1987, entre os casos (óbitos) obscrvamos que $52,9 \%$ apresentaram peso ao nascer menor que $2.500 \mathrm{~g}$; contra apenas $8,8 \%$ entre os controles (Tabcla 3). Essa distribuição resultou $\mathrm{em}$ RA de óbito de 11,63 para crianças nascidas com peso abaixo de $2.500 \mathrm{~g}$, em rclação às crianças que nasceram com peso considerado adequado. É um risco bastante
Tabela 3. Distribuição dos casos e controles, segundo o peso ao nascer, Botucatu-SP, 1987.

\begin{tabular}{|c|c|c|c|c|}
\hline \multirow{2}{*}{$\begin{array}{c}\text { Peso ao } \\
\text { nascer } \\
\text { (g) }\end{array}$} & Casos & Controles & \multirow[t]{2}{*}{$\mathrm{PA}$} & \multirow[t]{2}{*}{ IC } \\
\hline & $N^{2} \quad(\%)$ & Ne $(\%)$ & & \\
\hline$<2.500$ & $18(52,9)$ & $3(8,8)$ & 11,63 & $(3,40-39,81$ \\
\hline$\geq 2.500$ & $16(47,1)$ & $31(91,2)$ & & \\
\hline Total & $34^{*}(100)$ & $34^{*}(100)$ & & - \\
\hline $\begin{array}{ll}\text { RA } & - \text { Ris } \\
\text { IC } & - \text { Inte } \\
* & \text { Exo } \\
X^{2} & =15\end{array}$ & $\begin{array}{l}\text { Adicional } \\
\text { alo de Con } \\
\text { idas duas } \\
737 \text { ( } p<0,0\end{array}$ & $\begin{array}{l}\text { fiança } \\
\text { em inform } \\
\text { O1) }\end{array}$ & jes & \\
\hline
\end{tabular}

Tabela 4. Distribuição dos casos nascidos de gestação a termo e de seus controles, segundo o peso ao nascer, Botucatu-SP, 1987.

\begin{tabular}{|c|c|c|c|c|}
\hline $\begin{array}{c}\text { Peso ao } \\
\text { nascer }\end{array}$ & Casos & Controles & RA & IC \\
\hline (g) & $N^{8} \quad(\%)$ & No $\quad(\%)$ & & \\
\hline$<2.500$ & $9(37,5)$ & $2(8,3)$ & 6,60 & $(1,40-31,22)$ \\
\hline$\geq 2.500$ & $15(62,5)$ & $22(91,7)$ & & - \\
\hline Total & $24(100)$ & $24(100)$ & & \\
\hline
\end{tabular}

RA - Risco Adicional

IC - Intervalo de Confiança

$X^{2}=5,6595(p<0,02)$

Tabela 5. Distribuição dos casos nascidos de gestação pré-termo e de seus controles, segundo peso ao nascer, Botucatu-SP, 1987.

\begin{tabular}{|c|c|c|c|c|}
\hline \multirow{2}{*}{$\begin{array}{c}\text { Peso ao } \\
\text { nascer } \\
\text { (g) }\end{array}$} & Casos & Controles & \multirow[t]{2}{*}{ RA } & \multirow[t]{2}{*}{ IC } \\
\hline & $N^{2} \quad(\%)$ & $N^{a} \quad(\%)$ & & \\
\hline$<2.500$ & $9(81,8)$ & $1(9,1)$ & 45,0 & $(9,45-214,30)$ \\
\hline$\geq 2.500$ & $2(18,2)$ & $10(90,9)$ & & - \\
\hline Total & $11(100)$ & $11(100)$ & - & - \\
\hline $\begin{array}{ll}\text { RA } & - \text { Ris } \\
\text { IC } & - \text { Int } \\
X^{2} & =22\end{array}$ & $\begin{array}{l}\text { Adiciona } \\
\text { lo de Co } \\
76(p<0\end{array}$ & $\begin{array}{l}\text { ança } \\
\text { 1) }\end{array}$ & & \\
\hline
\end{tabular}

clevado se comparado com valores de outros estudos, todos ao redor de $4^{6,9,25}$. É possível tratar-se de uma questão de heterogencidade do grupo nascido com peso menor que $2.500 \mathrm{~g}$ no que concerne à idade gestacional. Com vista ao esclarecimento dessa hipótese procedeu-se ao controle dessa condição, "duração gestacional" (Tabclas 4 e 5). 
Ao se controlar a duração gestacional para o cálculo do RA de óbito provocado pelo peso alo nascer, observou-se uma importante discriminação: o RA de 11,63 passou agora para 6,60 entre os nascidos "a termo" e para 45,0 entre os nascidos "pré-termo". Este intervalo de variação do risco de morrer poderia ser auribuído mais à duração da gestação do que ao valor do peso ao nasecr, $\mathrm{cm}$ si mesmo. Porém, os valores médios do peso ao nascer de $1.350 \mathrm{~g}$ entre os prematuros c de $2.176 \mathrm{~g}$ entre os nascidos a termo mantêm o peso ao nascer como fator de risco de óbito. Estudo realizado $^{23}$ mostrou o papel da prematuridade $\mathrm{cm}$ relação à vitalidade e chances de sobrevida do recém-nascido, sendo o risco de óbito desta condição mais elevado do que o rclacionado à desnutrição intra-uterina de gestação a ıcrmo.

\section{Duração gestacional}

Como já se disse, hoje é indisculível a importância da prematuridade entre a mortalidade neonatal, seja devido à imaturidade pulmonar, seja devido à falta de defesas.

Trabalhos recentes apontam para a importância da prevenção de nascimentos prematuros na diminuição das taxas de mortalidade neonatal infan-

Tabela 6. Distribuição dos casos controles, segundo tempo de gestação, Botucatu-SP, 1987.

\begin{tabular}{lccccc}
\hline $\begin{array}{l}\text { Tempo de } \\
\text { gestação }\end{array}$ & Casos & Controles & RA & IC \\
& $N^{2}(\%)$ & $N^{Q}$ & $(\%)$ & & \\
\hline Pré-termo & $11(31,4)$ & $1(2,9)$ & 15,58 & $(2,82-86,09)$ \\
A termo & $24(68,6)$ & $34(97,1)$ & \\
Total & $35^{*}(100)$ & $35^{*}(100)$
\end{tabular}

RA - Risco Adicional

IC - Intervalo de Confiança

* Excluída uma sem informação

$x^{2}=9,9139(p<0,01)$

til $^{6,9,10}$. No presente trabalho observamos uma taxa de $2,9 \%$ de nascimentos prematuros (gestação prétermo) entre os controles, ou sobreviventes, enquanto entre os casos (crianças que foram a óbito) esta taxa foi de $31,4 \%$ (Tabela 6 ). O risco de morte para crianças nascidas prematuramente foi quinze vezes maior que para as nascidas a termo.

Em outros estudos realizados no Brasil ${ }^{9,25}$ esse risco não foi tão alto quanto cstc encontrado $\mathrm{cm}$ Botucatu, no ano de 1987. Vale ressaltar aqui a relação já mencionada entre os valores do peso ao nascer e a idade gestacional, que mostra menor valor médio de peso para os premaluros. Existe uma outra questão implícila nessa constatação: é a assistencial. Caberia saber a quantas andam os cuidados com os recém-nascidos prematuros em Botucalu, sendo que os dados indicam uma certa preciaricdade nesle particular. Caberia saber se se trala de precariedade na oferta ou no consumo da referida assistência.

\section{Escolaridade materna}

Encontrou-sc, para as crianças que foram a óbito, que $34,3 \%$ das suas mães apresentavam baixa escolaridade, enquanto para as crianças que sobreviveram essa taxa foi de 11,4\% (Tabela 7). $O$

Tabela 7. Distribuição dos casos e controles, segundo a escolaridade materna, Botucatu-SP, 1987.

\begin{tabular}{|c|c|c|c|c|c|c|}
\hline \multirow{2}{*}{$\begin{array}{c}\text { Escolaridade } \\
\text { Materna }\end{array}$} & \multicolumn{2}{|c|}{ Casos } & \multicolumn{2}{|c|}{ Controle } & \multirow[t]{2}{*}{ PA } & \multirow[t]{2}{*}{ IC } \\
\hline & $N^{Q}$ & $(\%)$ & $N^{8}$ & $(\%)$ & & \\
\hline$<4^{\mathrm{a}}$ Série & 12 & $(34,3)$ & 4 & $(11,4)$ & 4,04 & $(1,20-13,56)$ \\
\hline$\geq 4^{\mathrm{a}}$ Série & 23 & $(65,7)$ & 31 & $(88,6)$ & & \\
\hline Total & $35^{* *}$ & $(100)$ & $35^{\circ}$ & $*(100)$ & & \\
\hline
\end{tabular}

RA - Risco Adicional

IC - Intervalo de Confiança

* Inclui analfabetismo e instrução Mobral

- Excluida uma sem informação.

$X^{2}=5,1112(p<0,05)$

risco de morte entre as crianças de mães com baixa escolaridade foi quatro vezes maior que o risco observado entre as crianças de mães com escolaridade maior.

Estes resuludos são semelhantes ao da literatu$\mathrm{ra}^{9,25}$, os quais consideram a educação materna como lator importante para redução das taxas de mortalidade inläntil. Segundo a Investigação Interamericana ${ }^{22}$, em São Paulo, no pcríodo de 1968$70,54 \%$ das crianças falecidas no periodo pósneonatal cram fillhos de mães com escolaridade abaixo da cuarla séric do primciro grau, percentual maior que o encontrado nesse estudo. Essa diferença provavelmente rellete a variação do nível de escolaridade da população leminina $\mathrm{cm}$ duas conjunturas, espacial e temporalmente diferentes. No cntanto, apesar desta diferença, não se pode dizer que o risco de morrer medido por essa condição social cra também diferente, já que na cilada investigação não se consideraram os sobreviventes, não se calculando o referido risco. Este achado, no nosso 
entender, reflete menos a importância que a escolaridade em si pode ter para o cuidado das crianças e mais o significado da escolaridade como indicador de posição social e capacidade de consumo de bens e serviço. $O$ dado rcforça a impressão já expressa por outros autores de que a escolaridade é um sensivel indicador de condição social, cspecialmente em conjunturas urbanizadas como a presente ${ }^{20}$.

\section{Condiçōes de moradia}

Os dados de Tabela 8 mostram que $33,3 \%$ dos óbitos estudados apresentavam abastecimento de

Tabela 8. Distribuição dos casos e controles, segundo o tipo de abastecimento de água, Botucatu-SP, 1987.

\begin{tabular}{cccccccc}
\hline $\begin{array}{c}\text { Tipo de } \\
\text { abastecimento Casos } \\
\text { de água }\end{array}$ & No & \multicolumn{3}{l}{$\begin{array}{c}\text { Controle } \\
\text { (\%) }\end{array}$} & No & RA & IC \\
\hline Inadequado & 12 & $(33,3)$ & 2 & $(5,6)$ & 8,50 & $(2,06-35,1)$ \\
Adequado & 24 & $(66,7)$ & 34 & $(94,4)$ & - & \\
\hline Total & 36 & $(100)$ & 36 & $(100)$ &
\end{tabular}

R A - Risco Adicional
IC - Intervalo de Confiança
$X^{2}=8,7438(p<0,01)$

água inadequado, enquanto essa condição nos sobreviventes foi de somente 5,6\%. Encontramos um RA de morrer de 8,50 para as crianças com inadequação no abastecimento de água.

O que já se sabe da literatura é que existe forte associação entre óbilos gerados por doenças infccciosas e parasitárias (DIP) $\mathrm{e}$ as condições de sancamento ${ }^{24}$, estas expressas não só pclo abastecimento de água, mas também pelo destino dos dejetos. Porćm, já se viu que a participação das DIP foi irrisória como causa básica dos óbitos estudados, estes decorrentes em grande medida da prematuridade. Qual pode o ser o significado desta associação?

\section{Considerações finais}

O perfil de causas de óbito enconurado pclo presente estudo sugere a existência de dificuldades $c$ distorções no consumo de assistência pré-natal, ao parto e neonatal. Os elcvados RA relacionados à gestação pré-termo e ao peso ao nascer menor que $2.500 \mathrm{~g}$ configurariam a siluação causal imediatamente anterior ao óbito. As condições anteriores a este desfecho, particularmente aquelas ligadas à qualidade da assistência consumida durante a gestação, por não ter merecido a devida atenção na presente pesquisa, só podem ser disculidas por hipóteses, como se laz a seguir.

Os RA encontrados para baixa escolaridade materna e consumo inadequado de água, face ao perlï de mortalidade descrito, são de difícil explicação, pois aparentemente não existe nexo causal dircto entre essas duas condições e a prematuridade. A não ser que se considerem essas duas caractcrísticas familiares como componentes do mesmo perfil de reproducão social caracterizado no início destas conclusões. Scndo assim, as mesmas famílias que estariam solrendo dificuldades e distorções no consumo de assistência pré-natal, ao parto e neonatal, na conjuntura botucatuense de 1987, aprescntariam também as caractcrísticas de baixa escolaridade materna e consumo inadequado de água. Assim, ficaria melhor entendida a associação entre escolaridade c consumo de água com prematuridade, a principal causa dos óbitos.

Muito cmbora na fase inicial do presente trabalho tenha sido feito o levantamento preliminar dos óbitos e dos nascimentos de Bolucatu, cm anos anteriores a 1987, para se ter uma idćia da casuística a ser estudada, não foram consideradas as principais causas de óbito. Se isso tivesse sido feito, ter-sc-ia direcionado a claboração do questionário no sentido da obtenção de informações sobre condições e fatores sabidamente associados ao parto prematuro, ao baixo peso ao nascer c à mortalidade neonatal. Assim, tcríamos cvilado as limitações da análise dos resultados discutidas no parágrafo antcrior, particularmente no que se refere à qualidade da assistência consumida pelas mães e pclas crianças.

As perdas de óbitos sofridas pelo cstudo, cmbora tenha distribuição idêntica, do ponto de vista estulístico, ao grupo estudado, podem ter inlluído no resultado da associação entre classe social e óbito. As crianças da zona rural perdidas, provavelmente, se incorporariam ao subprolctariado, enquanto seus controles, ao proletariado. Isso resulLaria $\mathrm{cm}$ signilicância estatística. Sc assim fosse, ter-se-ia o principal indicador social do risco de óbito, condizente com o predomínio de prematuridade ao lado do inadequado consumo de instrução c de água, uma vez que seria a síntese das condições existenciais $\mathrm{cm}$ scu todo.

Em resumo, o perfil dos óbitos ocorridos nos menores de um ano de Bolucalu-SP, cm 1987, mostrou importantes desigualdades entre essas crianças com aquelas que sobreviveram, seja cm rclação ao peso ao nascer, scja $\mathrm{em}$ rclação à idade gestacional, scja ainda cm relação ao nível de instrução da mãe c à adequação do abastecimento domiciliar de água. Disto decorre que a assistência 
pré-natal e a pós-natal imediata deveriam capacitar-se melhor para atender os problemas identificados, bem como dedicar maior atenção às gestantes e seus recém-nascidos procedentes de famílias portadoras dos fatores de risco identilicados. Isto poderia evitar óbitos infantis decorrentes das causas verificadas. Seriam necessários lambém outros estudos que abordassem mais profundamentc os perfis de reprodução social das classes "subproletariado" e "proletariado propriamente dito"2, dominantes no presente estudo, nos quais fosse priorizada a assistência pré e perinatal. Parecc-nos que, em relação ao enfrentamento das desigualdades identificadas, a melhor estratégia residiria em ações político-econômicas que reduzissem as distâncias entre as classes sociais. Todas as familias deveriam ter a mesma capacidade de consumir os bens e serviços em igual quantidade $\mathrm{c}$ qualidade.

\section{Agradecimentos}

Ao Prof. Dr. Antonio Rufino Netto, pelas sugestões; à Rosana Teresa de Abrcu Dellevedove pelas entrevistas; ao Prof. Carlos Padovani, pela estatística.

MACHARELLI, C. A. \& OLIVEIRA, L.R. [Death risk profile of children under one year of age in an interior town of S. Paulo Stalc, Brazil, in 1987]. Rev. Saúde públ., S. Paulo, 25: 121-8, 1991. The infant mortality of children living in Botucatu, SP, Brazil, in 1987 is studied. In order to establish the risk profile for mortality, an additional risk factor (AR) was calculated on the basis some variables selected by means of a case control study. The results showed an AR for death of $15.58,11.63,8.50$ and 4.04 respectively for the groups of preterm gestation, low birth, absense of houschold water supply and insufficient maternal educational level, respectively. It was shown that there is a significant difference in socio-cconomic stalus between the families of survivors and those of nonsurvivors. This fact suggests that the best stralegy for overcoming the high infant mortality of the low income group is by improving their socio-econontic condition so that they can enjoy similar capacity to consume goods and services, in quantity and quality, as the high socio-cconomic group.

Keywords: Infant mortality. Risk. Sociocconomic factors.

\section{Referências Bibliográficas}

1. AMTGO, H.; MARQUES, N.; I3RITO, A.; SIQUEIRA, L. Mortalidade em menores de 5 anos na cidade de Recife-PE (Brasii): tendências $c$ associaçōes. Rev. Saúde públ., S. Paulo, 19: 531-42, 1985.
2. IBARROS, M. B. A. A utilização do conceito de classe social nos estudos dos perfis epidemiológicos: uma proposta. Rev. Saúde públ., S. Paulo, 20: 269-73, 1986.

3. BRIIILl1, 1. J. Epidemiologia: economia, medicina y polílica. Santo Domingo, Secretaria de Estado de Salud Pública y Assistencia Social, 1980.

4. BRLILII, J. \& GRANDA, E. Investigaçâo da saúde na sociedade:guia pedagógico sobre um novo enfoque do método epidemiológico. São Paulo, Instituto da Saúde/ABR ASCO, 1986.

5. BRIILH, J.; GRANDA, E.; CAMPANA, A.; BETANCOUR'T, $O$. Cuidad y muerte infantil. Quito, CEAS Ed., 1983.

6. CHESTER, L. G. C. Fatores de risco associados à mortalidade infantil cm Cotia e Vargem Grande Paulista, SP, 1984-1985; uma proposta de instrumentos preditivos. São Paulo, 1988. ['Tese de Doutaramento - Faculdade de Saúde Pública da USP!.

7. FISCIIMAN, A. \& GUIMARĀES, J. J. L. Risco de morrer no primciro ano de vida entre favelados e não favelados no Município de Porto Alegre, RS (Brasil) cm 1980. Rev. Saúde públ., S. Paulo, 20: 219-26, 1986.

8. GOODMAN, L. A. Simultaneous confidence intervals for contrasts among multinomial populations. Ann. Math. Stalist., 35: 716-25, 1964.

9. LACERIDA, M. V. Imporancia do peso ao nascer e outras variáveis na mortalidade pós-neonatal na região metropolitana do Rio de Janciro: análise de uma mostra de óbilos de maio a setembro de 1986. Rio de Janciro, Escola Nacional de Saúde Pública/ IIOCRUZ, 1989.

10. LAUREN'II, R.; BUCIALLA, C. M.; COSTA Jr.; M. L. Estudo da morbidade c montialide perinatal em matemidades segundo peso ao nasecr, idade da māe, assistência pré-natal e hábito de fumar da ināe. Rev. Saúde públ., S. Paulo, 19: 225-32, 1985.

11. L.ESIER, W. Crescimento da população na cidade de São l'aulo cutre 1950 e 1970 e scus rellexos nas condições de saúde pública. Cienc. e Cult., 27: 244-56, 1975.

12. MAC MAIION, B. \& PUGII, T. F. Epidemiology: principles and methods. Boston, Lille, Brown and Co., 1970.

13. MANUAL da Classificação Estatística Intemacional de Docnças, Lesôcs e Causas de Óbitos; $9^{2}$ revisāo, 1975. São Paulo, Contro da OMS para Classificação de Doenças em lortuguês/Ministério da Saúde/ Univesidade de São Paulo/Organização PanAmericana da Saúde, 1978. 2 v.

14. MARX, K. Salário, preço e lucro. $4^{2}$ ed. São Paulo, lìd. Glubal, 1985.

15. MIEIIINLN, O. S. Theoretical epidemiology. New York, Wiky Medical Publ., 1985.

16. MONTHIRO, C. A. I:stimativa de cocficientes específicos de mortalidade inlantil, segundo peso ao nascer no Município de São P'aulo (Brasil). Rev. Saúde públ., S. Paulo, 15: 603-10, 1981 .

17. MONTIIRO, C. A. Mortalidade inlantil e desenvolvimento social. Saúde Dab., (10): 27-9, 1985.

18. MON"IIIIRO, C. A. Saúde e nutriçäo das crianças de Säo Paulo: diagnóstico, contrastes sociais e tendências. São Paulo, Ld. Ilucitec, 1988.

19. MONITEIRO C. A.; BIENICIO, M. II. D' A.; BALDIJÃO, M. I. ^. Mortalidade no primeiro ano de vida e distribuição de renda c de recursos públicos de saúde. Rev. Saúde públ., S. P'aulo, 14: 515-39, 1980.

20. OLIVEIRA, L, R. de. Epidemiologia da desnutrição: tcoria e prática. São Paulo, 1989. ['Tese de Doutoramento - [raculdade de Saúde P'ública da USP]. 
21. OLIVEIRA, L. R.; RIZZATO, A. B. P.; MAG $A L D I, C$. Saúde matemo-infantil: visão crítica dos deterninantes e dos programas assistenciais. Rev. Saúde públ., S. Paulo, 17: 208-20,1983.

22. PUFFER, R. R. \& SERRANO, C. V. Caraclerísticas de la mortalidad en la niñez. Washington, D. C., Organización Panamericana de la Salud, 1973. (OPAS - P'ublicación Científica, 262).

23. SEMLNÁRIO Nacional de Aplicação do Enfoque de Risco nos Serviços de Saúde, Brasília, 1983. Manual de trcinamento. Brasília, DF, 1983. [Mimeografado]*.

* Os interessados poderão obter o trabatho diretamente com os autores do presente artigo.
24. VEITTER, D. M. \& SIMÕES, C. C. S. Acesso à infraestrutura de sancamento básico e mortalidade. Rev. bras. Listat., Rio de Janciro, 42(105) : 17-35, 1981.

25. VICTORA, C.G.; BARROS, I.C.; VAUGHAN, J.P. Epidemiologia da desigualdade. $2^{2}$ cd. São Paulo, Ed. Hucitce, 1989.

26. YUNES, J. \& RONCIIEZEL, V. S. C. Evolução da mortalidade geral, in fantil e proporcional no Brasil. Rev. Saúde públ., S. Paulo, 8(Supl.): 3-48, 1974.

Recebido para publicação em 24/8/1990 Reapresentado em 21/1/1991 Aprovado para publicação em $5 / 2 / 1991$ 